\title{
Rapid Measurement of Fatigue Behavior of Welded Joints Using the Lock-in Infrared Thermography
}

by Junling Fan*, Xinglin Guo*, Chengwei $\mathrm{Wu}^{*}$, Guojun $\mathrm{Ma}^{*}$

*Dept. of Engineering Mechanics, Dalian University of Technology, Dalian 116024, China, xlguo@dlut.edu.cn

\begin{abstract}
Considering the practical operating situations, the lock-in infrared thermography was used to rapidly determine the fatigue perfomance of welded joints subjected to a highly fixed mean stress. The results obtained by the lock-in infrared thermography were compared with the traditional results. Good predictions indicated the potential ability of the lock-in infrared thermography in investigating inhomogeneous welded joints. It is shown that the application of the lock-in infrared thermography as a non-destructive and non-contact method to predict fatigue behavior of materials and to detect the occurrence of fatigue damage for safety evaluation was suitable to consider the practical operating situations.
\end{abstract}

Key Words: Welded joints; Lock-in infrared thermography; fatigue behavior

\section{Introduction}

The application of the infrared thermography as a non-destructive and non-contact method to detect the occurrence of fatigue damage and to investigate the fatigue process of materials and components has become popular and has been widely and successfully studied over the last 20 years. From the experimental standpoint, the infrared thermography allows to measure the surface temperature of a specimen by means of an infrared thermal scanner and to monitor its growth during the test. On the basis of thermal experimental data, some analysis methods have been developed over the years in purpose of correlating the growing temperature to the physical processes of fatigue damage and failure in materials and components [1].

At present, four main evaluation methods for fatigue assessment are commonly used in practical applications: 1) Nominal stress method; 2) Hot-spot stress method; 3) Notch stress method; 4) Linear elastic fracture mechanics [2, 3]. The fatigue strength of welded joints is defined as the stress level at $2 \times 10^{6}$ cycles of the S-N curve in accordance with the current guidelines of the International Institute of Welding. It is based on the nominal stress and the hot-spot stress that the first two methods are able to be applied to determine fatigue parameters of welded joints. Nevertheless, they meet some unavoidable limitations in practical engineering designs [2]: the nominal stress is impossible to be decided in critical components with complex geometries; the selection of the fatigue strength is subjective due to the effects of the joint geometries and the loading histories on the fatigue responses; the hot-spot stress is significantly affected by the local stress at the welds with complex properties, which is strongly dependent on the finite element models; the size effect and the thickness effect are neglected during the analysis of the hot-spot stress method in order to simplify the processing procedures. The influence of the local weld on the fatigue responses is able to be taken into account by the notch stress method, and the parameterized formula is derived using the finite element analysis by idealizing the shape of the weld toe. It is sensitive to the crack size for the linear fracture mechanics method. The residual fatigue life of components with small cracks can be well predicted by use of the Paris theory, whereas the computational requirement is fairly greater than that needed in other methods. In addition, the fracture mechanics method is not suitable to determine the crack initiation life.

In the present paper, an application of the lock-in infrared thermographic methodology for predicting fatigue parameters of welded joints subjected to a high mean stress is presented to verify its validity and to extend its capabilities. 
The temperature evolution of the hot-spot zone on the welded joint surface during the fatigue test was used to analyze fatigue damage status related to safety evaluation. Resorting to the energy theory, an energetic damage model was established to predict the residual fatigue life of welded joints.

\section{Theoretical model}

\subsection{The lock-in infrared thermography}

From the analysis of high-cycle fatigue regime reported in the Refs. [2, 3, 4], it is observed that when a material or a component suffers from cyclic loading higher than its fatigue limit but lower than its yield limit, its surface temperature is well defined by three phases. The temperature rapidly rises in the initial stage (phase1), then tends to an asymptotic value after only a few cycles (phase 2), and finally it increases sharply due to the large plastic deformation before fatigue failure (phase 3). Among them, phases 1 and 2 are widely used to describe the fatigue damage status and to evaluate the fatigue parameters.

According to Fargione et al. [5], the fatigue limit is defined as the stress level for which the temperature $\Delta T$ increased, or the mean rate of temperature at the first stage, $\Delta T / \Delta N$, increased. As a consequence, the fatigue limit could be raplidly determined by plotting curves of the increase in temperature $\Delta T$, or the initial thermal gradient $\Delta T / \Delta N$, against the applied stress and finding value of the fatigue limit as the intercept of the curve on the $\mathrm{x}$-axis $(\Delta T=0$ or $\Delta T / \Delta N=0)$. After this, a number of attempts have been made to correlate the surface temperature increment to the fatigue parameter prediction [1, 3, 5]. In Refs. [2, 3, 6], a good correlation between the asymptotic temperature increment $\Delta T$ and the applied stress range squared $\Delta \sigma^{2}$ has been given experimentally and theoretically:

$$
\begin{cases}\Delta T_{S}=0 & \text { for } \Delta \sigma_{\mathrm{a}}<\Delta \sigma_{0} \\ \Delta T_{S}=A \cdot \Delta \sigma_{a}{ }^{2}+B & \text { for } \Delta \sigma_{\mathrm{a}} \leq \Delta \sigma_{0}\end{cases}
$$

where $\Delta T_{S}$ is the stable temperature increment; $\Delta \sigma_{\mathrm{a}}$ is the applied stress range; $\Delta \sigma_{0}$ is the fatigue limit; $A$ and $B$ are material constants.

With a set of specimens the successive stepwise loading procedure $[3,4]$ is applied on each of them at different stress range $\Delta \sigma$. The asymptotic temperature increments $\Delta T_{S}$ generated by the corresponding cyclic stresses are real-timely measured by use of an infrared thermal scanner. Finally, a regression line, $\Delta T_{S}$ versus $\Delta \sigma^{2}$, is drawn using the least-square method. Thus, the fatigue limit is rapidly determined by extrapolating the straight line down to zero at the $\mathrm{x}$-axis.

\subsection{The engergy approach}

From the energy dissipation standpoint, when the amount of the energy per unit volume reaches to a threshold value $E_{\mathrm{C}}$, fatigue failure will be occuring as soon as possible. $E_{\mathrm{C}}$ is a constant value that is independent of loading level and sequence, and that load interaction is not taken into account. Fargione et al. in Ref. [5] defined an energetic parameter $\Phi$, which enabled us to rapidly predict the whole S-N curve of materials and components with a limited number of specimens, theoretically only one specimen. $E_{C}[5]$ is documented to be proportional to the energetic parameter $\Phi$ calculated by integrating the $\Delta T-N$ curve recorded by a high-speed and high-sensitive infrared camera.

$$
E_{\mathrm{C}} \propto \Phi=\int_{0}^{N_{\mathrm{f}}} \Delta T(N) \cdot d N
$$


Due to the limited number of phase 1 and 3 mentioned above, the energetic parameter $\Phi$ can be simply expressed as:

$$
\Phi=\Delta T_{S} \cdot N_{\mathrm{f}}
$$

Using the successive stepwise loading procedure, the energetic parameter is easily determined resorting to the lock-in infrared thermography. To rapidly determine the fatigue behavior of materials and components, it is possible to obtain the asymptotic temeprature increment at each stress level, and then, based on eq. (3), to calculate the relevant $N_{\mathrm{f}}$. As a consequence, the entire S-N curve can be plotted based on the couples of $\left(\Delta \sigma, N_{\mathrm{f}}\right)$.

\subsection{The energetic damage model}

Based on the energy theory and the Miner's law, a linear energetic damage model was established below. The damage $D_{\mathrm{i}}$ of a material after $n_{\mathrm{i}}$ cycles under the cyclic stress $\sigma_{\mathrm{i}}$ is assumed to be:

$$
D_{\mathrm{i}}=\frac{n_{\mathrm{i}}}{N_{\mathrm{fi}}}
$$

where $N_{f}$ denotes the fatigue life under the stress amplitude $\sigma_{\mathrm{i}}$. As a consequence, the Miner's law is written as:

$$
D=\sum_{i=1}^{j} \frac{n_{\mathrm{i}}}{N_{\mathrm{fi}}}=1 \quad i=1,2,3, \cdots, j
$$

where $\mathrm{j}$ is the total number of stress levels.

Based on the energy dissipation theory, the damage accumulation rate can be replaced by the energy accumulation rate:

$$
D_{i}=\frac{n_{\mathrm{i}}}{N_{\mathrm{fi}}}=\frac{\Delta W_{\mathrm{i}} n_{i}}{E_{\mathrm{C}}}
$$

where $\Delta W_{\mathrm{i}}$ is the stable hysteresis energy.

In Ref. [6], Crupi presented the correlation between the stable hysteresis energy and the asymptotic temperature increment:

$$
\Delta W_{i}=\frac{\rho C_{p} \Delta T_{s i}}{N_{s i}}
$$

where $\rho$ is the material density; $C_{\mathrm{p}}$ is the specific heat capacity; $N_{\mathrm{si}}$ is the cycle number when the asymptotic temperature increase $\Delta T_{s i}$ is reached at the beginning of the fatigue test.

The limiting energy can be calculated by

$$
E_{c}=\Delta W_{i} \cdot N_{f i}=\frac{\rho C_{p} \Phi}{N_{s i}}
$$

Substituting Eqs. (7) and (8) into Eq.(6), the expression of the fatigue damage model is derived: 


$$
D_{i}=\frac{N_{i}}{N_{f i}}=\frac{\Delta T_{s i} \cdot N_{i}}{\Phi}
$$

Thus, the expression of the energetic damage model resorting to the energy theory can be written as:

$$
D=\sum_{i=1}^{k} \frac{\Delta T_{s i} \cdot N_{i}}{\Phi}=1 \quad i=1,2, \ldots, k
$$

In this paper, the cycle number $N_{\mathrm{i}}$ is equal to the fixed cycle number $N_{\mathrm{si}}$ after the surface temperature tends to be a stationary value. With the energy model above, the residual fatigue life can be predicted rapidly.

\section{Materials and methods}

\subsection{Material and specimen}

The material studied in the present research is FV520B steel, a type of martensite steels. It is widely used for mechanical components due to its high tensile strength of $1309 \mathrm{MPa}$ and a $0.2 \%$ offset yield strength of $1080 \mathrm{MPa}$ after a series of heat treatments as shown in Table 1. The main chemical composition of the steel is given in Refs. [3, 4]. The welded joints presented in Fig.1 were made using the manual arc-welding technique. Finally, the postweld heat treatment processes referring to the procedures in Table 1 were performed to eliminate the residual stress as a result of the welding process.

Table 1. Heat treatment processes

\begin{tabular}{|c|c|c|}
\hline Type of heat treatment & Conditions & Holding time \\
\hline Solution Treatment & $1050 \pm 10^{\circ} \mathrm{C}$, air-cooling & $1.5-2.5 \mathrm{~h}$ \\
\hline Thermal Refining Treatment & $850 \pm 10^{\circ} \mathrm{C}$, oil-cooling & $1.5-2.5 \mathrm{~h}$ \\
\hline Aging Treatment & $480 \pm 10^{\circ} \mathrm{C}$, air-cooling & $2.0-3.0 \mathrm{~h}$ \\
\hline
\end{tabular}

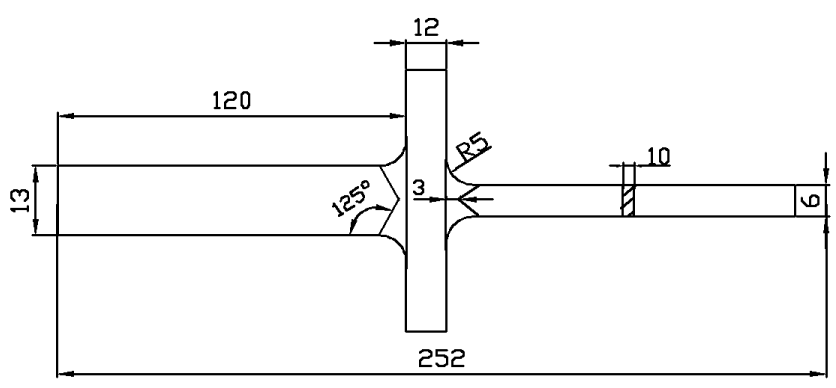

Fig.1. The welded joint (unit: $\mathrm{mm}$ )

\subsection{Methods}

Tests were carried out using a MTS810 servo-hydraulic machine with a $100 \mathrm{kN}$ capacity. The present fatigue tests were different from the previous work due to the highly fixed mean stress level $\sigma_{\mathrm{m}}=640 \mathrm{MPa}$ considering the practical situations. The nominal stress amplitudes in a sinusoidal waveform are in the range of 80 200 MPa. The fatigue test was terminated to install the next specimen if fracture did not occur within 5 million cycles. To attain reliable data for a statistical analysis, 32 specimens were tested in turn. 
Moreover, using the lock-in infrared thermography, tests at increasing loads were carried out by a stepwise succession, starting from $140 \mathrm{MPa}$ with steps of $20 \mathrm{MPa}$ every 60000 cycles. The surface temperature of the tested specimens was recorded by the Cedip Jade III infrared scanner with a $320 \times 240$ pixels focal-plane-array InSb detector.

\section{Results and discussion}

\subsection{Traditional fatigue results}

Fig.2 is the S-N curve in bi-logarithm scale by fitting the fatigue data using the least-square method. Fatigue life was assumed to be the dependent variable in the S-N tests, whereas the stress range was set as the independent variable. The fatigue strength (fatigue class, FAT) and the fatigue limit were determined by extrapolating the S-N curve down to 2 and 5 million cycles, respectively. To consider the safety, the S-N curve with a higher survival probability of $97.7 \%$ is also shown in Fig.2. It is observed that both the fatigue strength and the fatigue limit for the S-N curve with $97.7 \%$ survival probability are lower than that of the median S-N curve ( $50 \%$ survival probability). The higher the survival probability, the lower the fatigue strength and the fatigue limit.

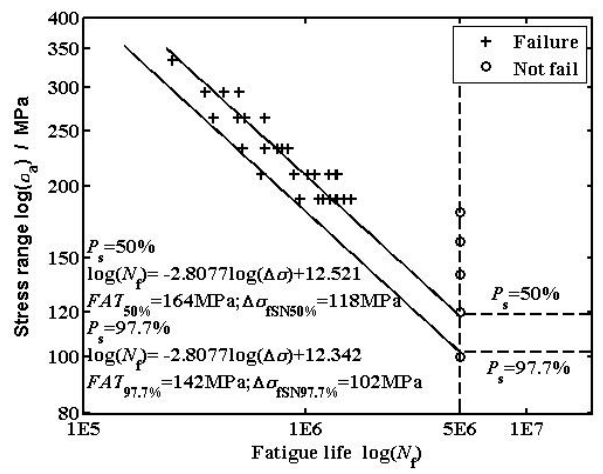

Fig.2. Fatigue S-N curves

\subsection{Predicted fatigue results}

During the fatigue tests, the temperature $T(t)$ of the specimen surface and the environmental temperature $T_{0}$ were real-timely detected by the infrared camera. Here the differential thermographic technique was applied to calculate the surface temperature increment $\Delta T_{S}$ for the purpose of avoiding any errors resulted from the environmental perturbation and the limited sensitivity of the experimental system.

$$
\Delta T_{\mathrm{s}}=T(t)-T_{0}
$$

The detailed process of the differential thermographic method is schematically presented in Fig.3. Resorting to the stepwise loading procedure, all the stationary temperature increase $\Delta T_{\mathrm{S}}$ corresponding to the applied stress level $\sigma$ is able to be determined using the thermographic method mentioned above.

Fig. 4 shows the $\Delta T-N$ curves at different nominal stress amplitudes $\sigma_{\mathrm{ai}}$. The surface temperature is almost stationary before the first 40000 cycles negligible compared with the whole fatigue life. It is obviously observed that the $\Delta T-N$ curves take on typical three phases.

Fig. 5 shows the $\Delta T-N$ curve of a welded joint tested at an increasing stepwise loading. Fig. 6 presents the thermographic images of the hottest zone on the specimen surface at increasing cyclic loading during a successive loading procedure. The physical evolution of the specimen is able to be identified by the thermographic images presented in Fig.6. It is observed that the hot-spot zone expanded as the applied stress increased, demonstrating a strongly 
localized fatigue damage phenomenon induced by the localized stress concentration. Once the threshold value of fatigue damage was reached, fatigue fracture would take place at the hot-spot zone due to its complex material properties and stress state.
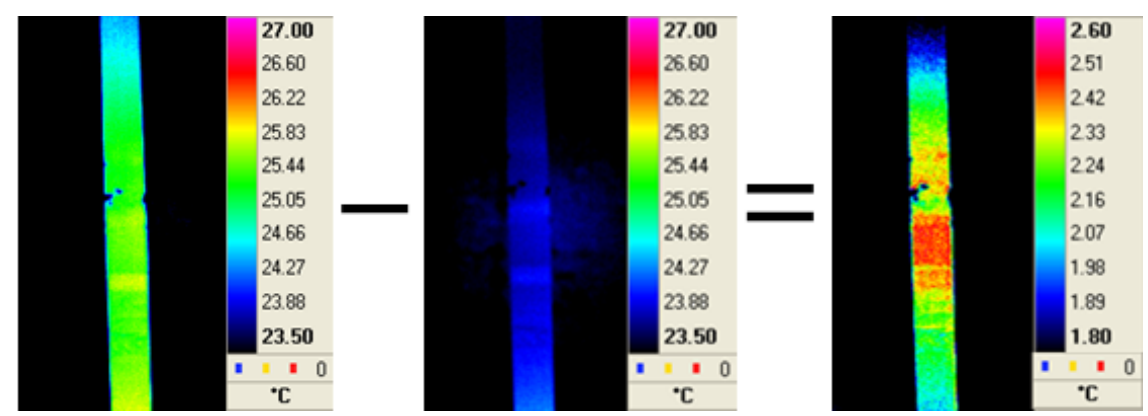

Fig.3. The differential thermographic method

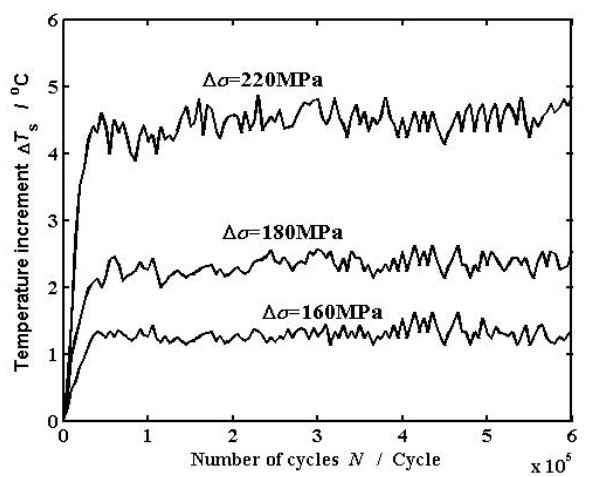

Fig.4. $\Delta T-N$ curves

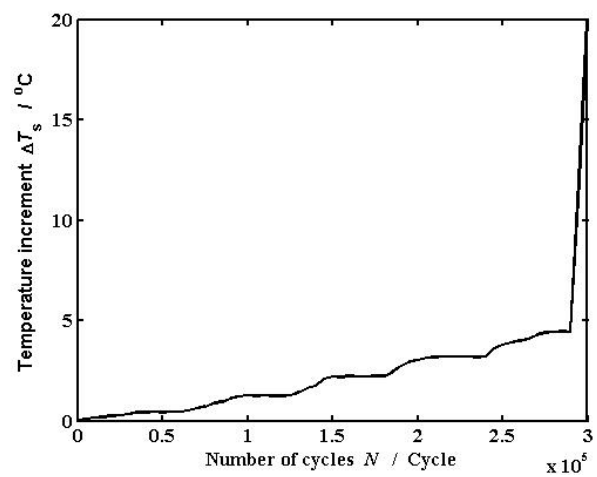

Fig.5. $\Delta T-N$ curve at an increasing laoding procedure

(c)

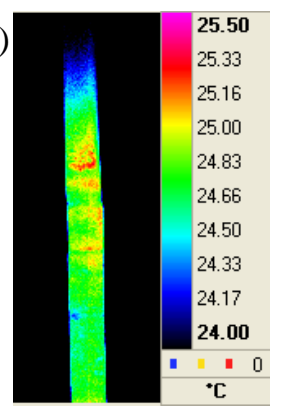

(d)

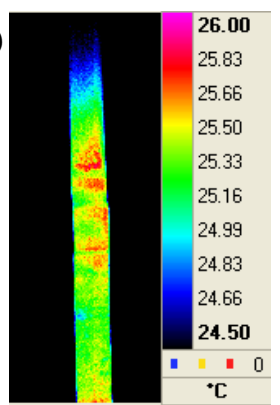

Fig.6. Hot-spot zone at different nominal stress ranges:

(a) $140 \mathrm{MPa}$; (b) $160 \mathrm{MPa}$; (c) $180 \mathrm{MPa}$; (d) 200MPa

The thermographic data, i.e. the stationary temperature increment $\Delta T_{\mathrm{S}}$, was directly associated with the corresponding stress range squared $\Delta \sigma^{2}$. A regression line presented in Fig.7 using the least-square method was readily plotted based on the couples of $\left(\Delta T_{S}, \Delta \sigma^{2}\right)$. As a consequence, the fatigue limit was determined as $123 \mathrm{MPa}$ by extrapolating the regression line down to zero at the $\Delta \sigma^{2}$-axis.

Fig.8 shows the whole S-N curves at different survival probabilities using the energy approach. It is clearly shown that the material constants $m=2.9021$ and $C=12.6559$ are in good agreement with the ones given in Fig. 2 obtained by the traditional testing method. To demonstrate the good predictions of the infrared thermographic methodology, the fatigue parameters $\Delta \sigma_{\mathrm{fSN}}$ obtained by the traditional procedures were compared with the predicted values $\Delta \sigma_{\mathrm{fp}}$ assessed by the 
infrared thermography and the energy approach. Table 2 is the comparison between $\Delta \sigma_{\mathrm{fSN}}$ and $\Delta \sigma_{\mathrm{fp}}$. Note that the errors between the predicted values and the traditional results are less than $10 \%$. It is concluded that the infrared thermography enables us to well predict the fatigue parameters of welded joints subjected to fatigue loading even though a high mean stress level $\sigma_{\mathrm{m}}=640 \mathrm{MPa}$ is applied on the welded components.

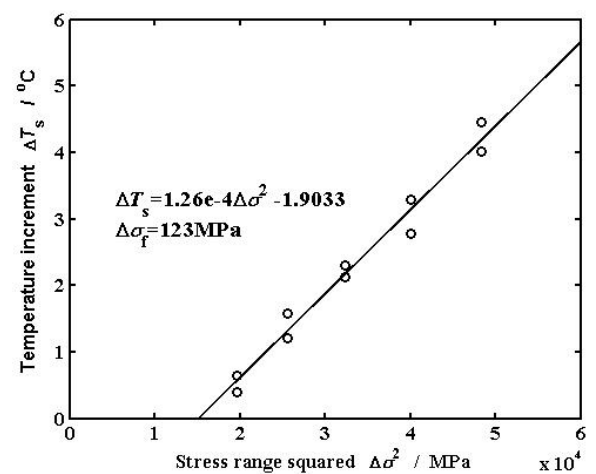

Fig.7. The S-N curve by the infrared thermography

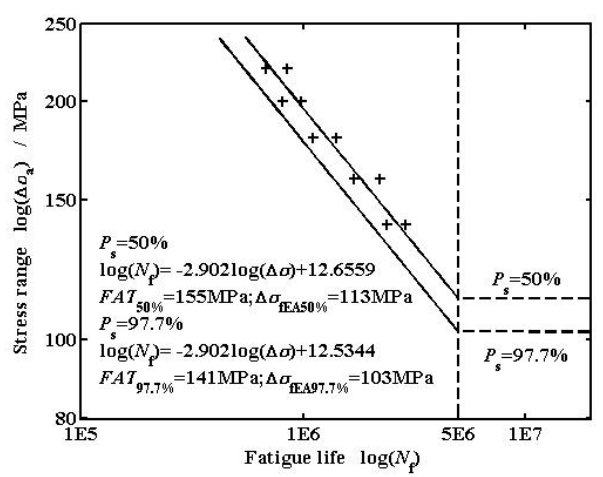

Fig.8. S-N curves by the energy approach

Table 2. Comparison of the fatigue parameters $\left(P_{s}=50 \%\right)$

\begin{tabular}{|c|c|c|c|c|}
\hline Method & FAT / MPa & $\delta \%$ & Fatigue limit $\sigma_{\mathrm{f}} / \mathrm{MPa}$ & $\delta \%$ \\
\hline The traditional method & 164 & -- & 118 & -- \\
\hline The energy approach & 155 & $5.5 \%$ & 113 & $4.2 \%$ \\
\hline The infrared methodology & -- & -- & 123 & $4.2 \%$ \\
\hline
\end{tabular}

\subsection{Residual fatigue life prediction}

In this part, two welded specimens, undergoing two cyclic stress levels with different loading sequences, were tested to confirm the capability of the energetic damage model in predicting the residual fatigue life. The two sinusoidal stress ranges were, respectively, $180 \mathrm{MPa}$ and $220 \mathrm{MPa}$, and the surface temperature increments were real-timely recorded by an infrared camera. Table 3 presents the detailed testing processes. The errors between the predicted values by the energetic damage model and the actual values are less than $15 \%$, achieving good predictions. That confirms that the fatigue responses of materials and components are more or less affected by the loading sequences. The possible reason is that the crack density rapidly increases under the high-low loading sequence. By contrast, this phenomenon is not obviously observed. It is concluded that the energetic damage model not only gives accurate predictions of the residual fatigue life, but also presents a clear physical meaning from the view of the energy accumulation.

Table 3. Comparison between the energetic damage model $\left(P_{s}=50 \%\right)$

\begin{tabular}{ccccccccc}
\hline & $\sigma_{\mathrm{a} 1} / \mathrm{MPa}$ & $\Delta T_{\mathrm{s} 1} /{ }^{\circ} \mathrm{C}$ & Cycles $N_{1}$ & $\sigma_{\mathrm{a} 2} / \mathrm{MPa}$ & $\Delta T_{\mathrm{s} 2} /{ }^{\circ} \mathrm{C}$ & Predicted life & Actual life & Errors \\
\hline$\# 1$ & 180 & 1.95 & 450000 & 220 & 3.89 & 335100 & 378296 & $11.4 \%$ \\
$\# 2$ & 220 & 3.73 & 300000 & 180 & 2.07 & 513062 & 597431 & $14.1 \%$ \\
\hline
\end{tabular}

\section{Conclusions}

(1) The lock-in infrared thermography, as a mature technique, enables us to rapidly evaluate the fatigue behavior of inhomogeneous welded joints, such as, fatigue limit and the whole S-N curve, with a limited number of specimens. Good predictions are presented even though a high mean stress is applied to take into account the practical situations during the fatigue tests. 
(2) The hottest zone on the specimen surface is the critical location related to the final fatigue fracture. The temperature field monitored by the infrared thermal scanner is able to be used to assess the fatigue damage status for safety evaluation.

(3) The energetic parameter $\Phi$, independent of the loading sequences, is a constant value related to material properties even though a high mean stress is applied. On the basis of the limiting energy $\mathrm{E}_{\mathrm{C}}$ proportional to the energetic parameter $\Phi$, the residual fatigue life of the welded joint is able to be determined, and a good prediction is confirmed with the actual value. The energetic damage model exhibits a clear physical meaning from the view of the energy accumulation during the fatigue process.

\section{Acknowledgements}

The financial supports of Natural Science Foundation of China (No.11072045) and the National Basic Research Program of China (2011CB706504) are gratefully acknowledged.

\section{REFERENCES}

[1] Curà F., Curti G., Sesana R.., "A new iteration method for the thermographic determination of fatigue limit in steels". Int. J. Fatigue, vol. 27, pp. 453-459, 2005.

[2] Fan J.L., Guo X.L., Zhao Y.G., Wu C.W., "Predictions of S-N curve and residual life of welded joints by quantitative thermographic method". J. Mater. Eng., vol. 12, pp. 29-33, 2011. (in Chinese)

[3] Fan J.L., Guo X.L., Wu C.W., Zhao Y.G., "Research on fatigue behavior evaluation and fatigue fracture mechanisms of cruciform welded joints". Mater. Sci. Eng. A, vol. 528, pp. 8417-8427, 2011.

[4] Fan J.L., Guo X.L., Wu C.W., Deng D.W., "Effect of heat treatments on fatigue properties of FV520B steel using infrared thermography". Chin. J. Mater. Res., vol. 26, pp. 61-67, 2012. (in Chinese)

[5] Fargione G., Geraci A., La Rosa G., Risitano A., "Rapid determination of the fatigue curve by the thermographic method". Int. J. Fatigue, vol. 24, pp. 11-19, 2002.

[6] Crupi V., Guglielmino E., Maestro M., Marino A., "Fatigue analysis of butt welded AH36 steel joints: Thermographic Method and design S-N curve". Mar. Struct., vol. 22, pp. 373-386, 2009. 